\title{
The use of glucocorticoids in rheumatoid arthritis - no'rational' approach yet
}

\author{
Sonali P Desai* and Daniel H Solomon \\ See related research by Ibanez et al., http://arthritis-research.com/content/12/2/R50
}

\begin{abstract}
The relationship between glucocorticoids and bone mineral density in rheumatoid arthritis is complex. Further study into the optimal dosing, timing and duration of glucocorticoid use in rheumatoid arthritis is necessary.
\end{abstract}

In the previous issue of Arthritis Research and Therapy, Ibanez and colleagues [1] report on the 'rational' use of glucocorticoids (GCs) in the management of early arthritis. This article concludes that GCs cause minimal variation in bone mineral density (BMD) at multiple skeletal sites, and in fact may increase BMD at the ultradistal forearm, a juxta-articular site. Although this article is notable for examining the effect of GCs on BMD at five anatomic sites, a 'rational' use of GCs for rheumatoid arthritis (RA) is still elusive. To grasp the complex relationship between GCs and both localized (juxta-articular, bony erosions) and systemic (osteoporosis) bone loss in RA, we need to first step back and appreciate the interplay of the immune system and bone metabolism.

The osteoclast plays a central role at the site of inflamed joints and is critical in the pathogenesis of joint erosions in RA [2]. Receptor activator of nuclear factor-kappa B ligand (RANKL), expressed by TH1 and TH17 T cell subsets, is a potent inducer of osteoclast differentiation. Additionally, an array of pro-inflammatory cytokines such as TNF, IL-1, IL-6 and IL-17 can stimulate RANKL expression [3] (Figure 1). GCs, in turn, directly affect both osteoblast and osteoclast activity, and indirectly exert many effects on bone metabolism, leading to an increased fracture risk [4] (Figure 2).

How should the relationship between the bone biology in RA and the effects of GCs translate into the use of GCs in clinical practice? The COBRA trial provides a rationale

*Correspondence: sdesai5@partners.org

Brigham and Women's Hospital, Division of Rheumatology, Immunology, and Allergy, 75 Francis Street, Boston, MA 02115, USA for the use of GCs in combination with other disease modifying anti-rheumatic drugs (DMARDs) to significantly reduce RA disease activity [5]. A subsequent review demonstrates that GCs (mean cumulative dose of 2,300 mg prednisone equivalent over the first year), when used in combination with traditional DMARD therapy, can decrease the rate of radiographic progression in RA [6]. The effect of GCs on bone mass, among non-RA patients, has been evaluated in a small randomized, placebo-controlled trial demonstrating that serum markers of bone formation are rapidly decreased among healthy post-menopausal women treated with just $5 \mathrm{mg}$ of prednisone daily for 6 weeks [7]. In early RA patients treated with prednisolone $7.5 \mathrm{mg}$ per day and traditional DMARD therapy (compared to traditional DMARD therapy alone), markers of bone formation, markers of bone resorption, and lumber spine BMD, but not femoral BMD, were decreased [8]. However, in a randomized, placebo-controlled trial of 95 early RA patients, GCs decreased the degree of localized hand bone loss [9]. These studies suggest that low dose GCs may reduce markers of bone formation leading to generalized osteoporosis, but they also counteract RA-associated inflammation and slow the rate of bone loss proximal to sites of active disease. Other factors, such as GC resistance and genetic polymorphisms that predispose to either GC sensitivity or resistance, may play a role in the BMD variation seen at various anatomic sites $[10,11]$.

Despite the disease-modifying properties of GCs seen in RA patients, the risk of GC-induced osteoporosis and its associated morbidity often give the rheumatologist pause when determining whether to use GCs, in what dosing, for how long, at what time in the disease process, and in which types of RA patients (seropositive versus seronegative). Ibanez and colleagues have begun to advance our knowledge of GC use in RA. In this cohort of early RA patients, they found a significant decrease in BMD at all sites except the ultradistal and distal forearm. In the multivariate analysis there was no significant relationship between cumulative GC use and BMD variation at multiple sites, except at the ultradistal (increased BMD) and mid-forearm (decreased BMD) [1]. 


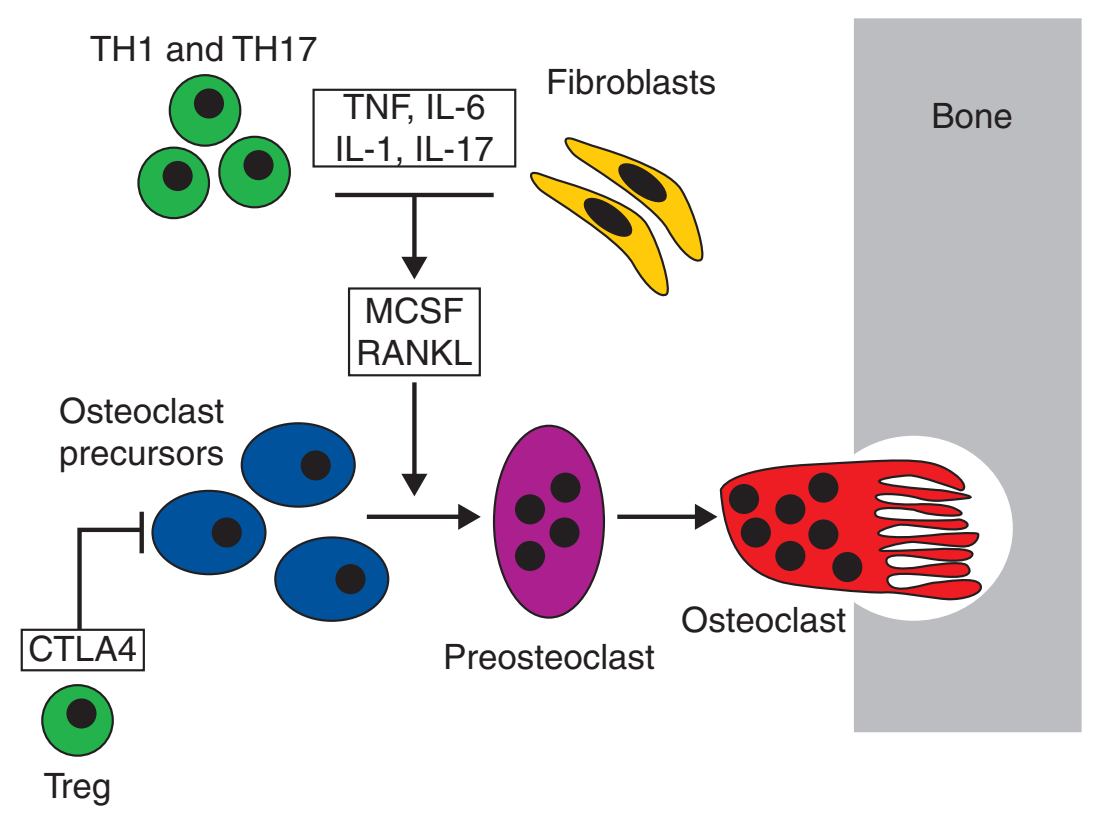

Figure 1. Osteoclast formation in the joint. Monocytic cells in the synovium serve as osteoclast precursors. Upon exposure to macrophage colonystimulating factor (MCSF) and Receptor activator of nuclear factor-kappa B ligand (RANKL) synthesized by T cells and synovial fibroblasts, osteoclasts fuse to polykaryons termed preosteoclasts, which then undergo further differentiation into mature osteoclasts, acquiring specific features such as the ruffled membrane. Inflammatory cytokines such as TNF and IL-1, IL-6, and IL-17 increase the expression of RANKL and thus support osteoclastogenesis in the joint. In contrast, regulatory T cells (Tregs) block osteoclast formation via Cytotoxic T-lymphocyte antigen 4 (CTLA4). Figure obtained with permission from [3]. The full colour version of this figure is available online at http://arthritis-research.com/content/12/3/127

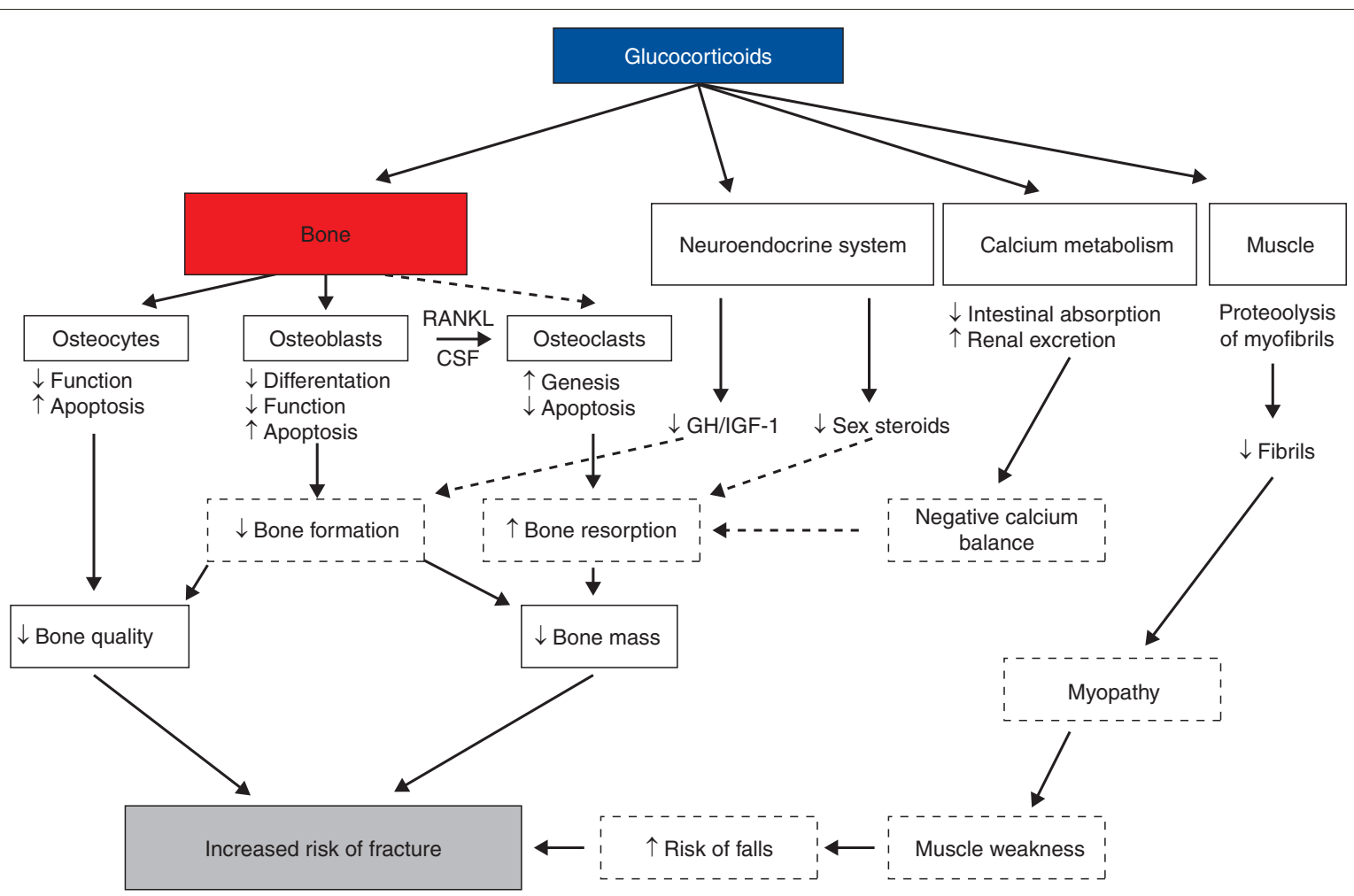

Figure 2. The direct and indirect effects of glucocorticoids on bone leading to glucocorticoid-induced osteoporosis and fractures. Figure obtained with permission from [4]. CSF, colony stimulating factor; GH, growth hormone; IGF, insulin-like growth factor; RANKL, Receptor activator of nuclear factor-kappa B ligand. The full colour version of this figure is available online at http://arthritis-research.com/content/12/3/127 
At first glance, the seemingly simple lack of BMD variation among a cohort of early $\mathrm{RA}$ patients treated with GCs is a compelling argument for GC use - but in this small 2-year study of early RA, the dosing, duration and long-term effects remain unknown. The apparent increase in ultradistal forearm BMD among patients treated with GCs warrants further exploration. However, this study of early RA examines only 116 patients treated with a median cumulative GC dose of $22 \mathrm{mg} / \mathrm{month}$ and $45 \mathrm{mg} / \mathrm{month}$ among those who actually received GCs. Is it the relatively small cumulative dose of GCs used in this study that explains the lack of significant BMD variation or the fact that only $67 \%$ of the cohort actually received GCs, with $17.3 \%$ of patients on GC therapy at the end of the study?

\section{Conclusion}

While Ibanez and colleagues have furthered our understanding of the effects of GCs on BMD variation through their detailed analysis of five skeletal sites, we are far from a 'rational' use of GCs in the management of RA.

\section{Abbreviations}

$\mathrm{BMD}=$ bone mineral density; $\mathrm{DMARD}=$ disease modifying anti-rheumatic drug; $G C=$ glucocorticoid; $I L$ = interleukin; RA = rheumatoid arthritis; RANKL

$=$ Receptor activator of nuclear factor-kappa B ligand; TNF = tumor necrosis factor.

\section{Competing interests}

The authors declare that they have no competing interests.

\section{Acknowledgements}

SPD's effort is supported by the American College of Rheumatology Research and Education Fund Physician Scientist Development Award. DHS's effort is supported by NIH grants (AR 055989 and AR 047782).

Published: 25 June 2010

\section{References}

1. Ibanez M, Ortiz AM, Castrejon I, Garcia-Vadillo A, Carvajal I, Castaneda S, Gonzalez-Alvaro I: A rational use of glucocorticoids in patients with early arthritis has a minimal impact on bone mass. Arthritis Res Ther 12:R50.

2. Gravallese EM, Harada Y, Wang JT, Gorn AH, Thornhill TS, Goldring SR: Identification of cell types responsible for bone resorption in rheumatoid arthritis and juvenile rheumatoid arthritis. Am J Pathol 1998, 152:943-951.

3. Schett G: Osteoimmunology in rheumatic diseases. Arthritis Res Ther 2009, $11: 210$.

4. Canalis E, Mazziotti G, Giustina A, Bilezikian JP: Glucocorticoid-induced osteoporosis: pathophysiology and therapy. Osteoporos Int 2007, 18:1319-1328.

5. Maurice MM, Nakamura H, van der Voort EA, van Vliet Al, Staal FJ, Tak PP, Breedveld FC, Boers M, Verhoeven AC, Markusse HM, van de Laar MA, Westhovens R, van Denderen JC, van Zeben D, Dijkmans BA, Peeters AJ, Jacobs P, van den Brink HR, Schouten HJ, van der Heijde DM, Boonen A, van der Linden S: Randomised comparison of combined step-down prednisolone, methotrexate and sulphasalazine with sulphasalazine alone in early rheumatoid arthritis. Lancet 1997, 350:309-318.

6. Kirwan JR, Bijlsma JW, Boers M, Shea BJ: Effects of glucocorticoids on radiological progression in rheumatoid arthritis. Cochrane Database Syst Rev 2007:CD006356.

7. Ton FN, Gunawardene SC, Lee H, Neer RM: Effects of low-dose prednisone on bone metabolism. J Bone Miner Res 2005, 20:464-470.

8. Engvall IL, Svensson B, Tengstrand B, Brismar K, Hafstrom I: Impact of lowdose prednisolone on bone synthesis and resorption in early rheumatoid arthritis: experiences from a two-year randomized study. Arthritis Res Ther 2008, 10:R128.

9. Haugeberg G, Strand A, Kvien TK, Kirwan JR: Reduced loss of hand bone density with prednisolone in early rheumatoid arthritis: results from a randomized placebo-controlled trial. Arch Int Med 2005, 165:1293-1297.

10. Sliwinska-Stanczyk P, Pazdur J, Ziolkowska M, Jaworski J, KaminskaTchorzewska E, Lacki JK: The effect of methylprednisolone on proliferation of PBMCs obtained from steroid-sensitive and steroid-resistant rheumatoid arthritis patients. Scand J Rheumato/ 2007, 36:167-171.

11. Manenschijn L, van den Akker EL, Lamberts SW, van Rossum EF: Clinical features associated with glucocorticoid receptor polymorphisms. An overview. Ann N Y Acad Sci 2009, 1179:179-198.

doi:10.1186/ar3035

Cite this article as: Desai SP, Solomon DH: The use of glucocorticoids in rheumatoid arthritis - no'rational' approach yet. Arthritis Research \& Therapy 2010, 12:127. 